\title{
The crystal structure of recombinant Lactococcus lactis prolidase
}

\author{
Pawel Grochulski $^{a}$, Oarabile Kgosisejo ${ }^{b}$, Jian An Chen ${ }^{b}$, and Takuji Tanaka \\ ${ }^{a}$ Canadian Light Source Inc., 44 Innovation Boulevard Saskatoon, SK, S7N 2V3 \\ Canada, ${ }^{b}$ Department of Food and Bioproduct Sciences College of Agriculture and Bioresources \\ University of Saskatchewan, 51 Campus Drive, Saskatoon, SK S7N 5A8 \\ Canada
}

Lactococcus lactis prolidase (Xaa-Pro dipeptidase; EC.3.4.13.9) is unique from other prolidases by showing allosteric behaviour, substrate inhibition, and metal-dependent substrate specificity. Its substrate preference also shifts from Leu - Pro to Arg - Pro when zinc in the reaction mixture is replaced by manganese. In this present study, the three-dimensional structure of recombinant L. lactis prolidase was determined by X-ray crystallography at $2.25 \AA$ resolution. Three molecules are located in the crystal asymmetric unit where molecule A forms a dimer with molecule $\mathrm{B}$, while molecule $\mathrm{C}$ forms a dimer with molecule $\mathrm{C}^{\prime}$ in the adjacent asymmetric unit. Of all the three molecules, molecule $\mathrm{C}$ is less defined and incomplete. While this fact compromises the overall quality of the refined model, the functional interpretation of the structure is not compromised since the biologically-functional homodimeric configuration of $L$. lactis prolidase is represented by well-defined molecules A and B. The refined model confirmed that there is a twelve-residue (residues 32 - 43) loop structure from one subunit over the active site of the other subunit, proving the existence of the putative loop structure in our previous study. This loop is three amino acids longer than the loops of prolidases of Pyrococcus furious (1pv9) and Pyrococcus horikoshii OT3 (1wy2). The crystal structure shows the loop structure can form two states ("open" and "closed" states) through interaction between the loop and active site proximity. It supports the proposed formation of allosteric site by the loop and Arg 293. 